\title{
Methicillin-Resistant Staphylococcus aureus (MRSA)-Positive Pericardial Abscess Presenting in a Hemodialysis Patient
}

\author{
Christopher C. Zarour ${ }^{1}$, Mario Dervishi ${ }^{2}$, Daniel Fuguet ${ }^{1}$, Zahraa Al-bahbahanee ${ }^{3}$ \\ 1. Radiology, St. Joseph Mercy Hospital, Pontiac, USA 2. Radiology, American University of the Caribbean School of \\ Medicine, Cupecoy, SXM 3. Internal Medicine, University of Baghdad Al-Kindy College of Medicine, Baghdad, IRQ
}

Corresponding author: Christopher C. Zarour, christopher.zarour@stjoeshealth.org

\begin{abstract}
Purulent pericarditis is an uncommon infection of the pericardial space that can very rarely present as a pericardial abscess. Infection by hematogenous spread in dialysis patients is among the predisposing risk factors that can lead to purulent pericarditis. Herein, we present a pericardial abscess case due to methicillin-resistant Staphylococcus aureus (MRSA) infection in a male patient with end-stage renal disease (ESRD) on hemodialysis. The patient has a past surgical history of two prior pericardial effusions due to uremic pericarditis.
\end{abstract}

Categories: Internal Medicine, Radiology, Infectious Disease

Keywords: pericardial abscess, pericardial cyst, methicillin-resistant staphylococcus aureus, end-stage renal disease.

\section{Introduction}

Purulent pericarditis is an extremely rare infectious etiology affecting the pericardial space characterized by gross pus in the pericardium. It is mostly associated with nosocomial hematogenous spread, as can be seen in dialysis or surgical interventions such as pericardial windows [1].

Predisposing risk factors of purulent pericarditis include pericardial effusion (infectious or inflammatory), chronic kidney disease, immunocompromised status, alcohol abuse, cardiothoracic surgery, and trauma [1]. It has been revealed that patients with purulent pericarditis also had preexisting pericardial diseases as a result of uremia, malignancy, or collagen vascular diseases [1,2].

Purulent pericarditis as an acute illness is characterized by high fever, tachycardia, cough, and chest pain. However, rarely no signs of sepsis of may be appreciated, which makes the diagnosis extremely difficult [3]. Infection usually involves the entire pericardium, but can rarely present as a pericardial abscess [4].

Received 08/23/2020

Review began 09/08/2020 Review ended 09/09/2020 Published 09/12/2020

๑) Copyright 2020

Zarour et al. This is an open access article distributed under the terms of the Creative Commons Attribution License CC-BY 4.0., which permits unrestricted use, distribution, and reproduction in any medium, provided the original author and source are credited.
Two-dimensional echocardiography and CT are crucial in early detection to decrease mortality [4]. However, there are many mimics that may resemble pericardial abscess, and as a result imaging cannot be used alone. Diagnosis must be established by image-guided needle aspiration of the fluid collection in the pericardium, with appropriate fluid cytology and culture, to ensure the offending etiology is pinpointed and immediate therapy is initiated.

\section{Case Presentation}

A 42-year-old male with a past medical history of end-stage renal disease (ESRD) on hemodialysis presented to the emergency department (ED) with severe anemia and hypotension after hemodialysis.

On physical examination, the patient appeared in no acute distress, while denying fever, chills, nausea, vomiting, or excessive weight loss. His temperature was elevated at 101.2 degrees Fahrenheit. Lab values were unremarkable. With a past history of two pericardial windows attributed to uremic pericarditis/pericardial effusion, a CT of the chest, abdomen and pelvis with intravenous and oral contrast was performed (Figures 1-3). The CT demonstrated a thick-walled right cardiophrenic angle cystic mass measuring 9 x 11 x $11 \mathrm{~cm}$ (anteroposterior x transverse x craniocaudal dimensions) causing mass effect on the right atrium and ventricle. An emergent interventional radiology consultation was placed, and a CTguided needle aspiration was performed. Fluid analysis and culture yielded positive methicillin-resistant Staphylococcus aureus, in keeping with a pericardial abscess. The pericardial abscess was drained and treatment with vancomycin ensured a complete recovery. 


\section{Cureus}

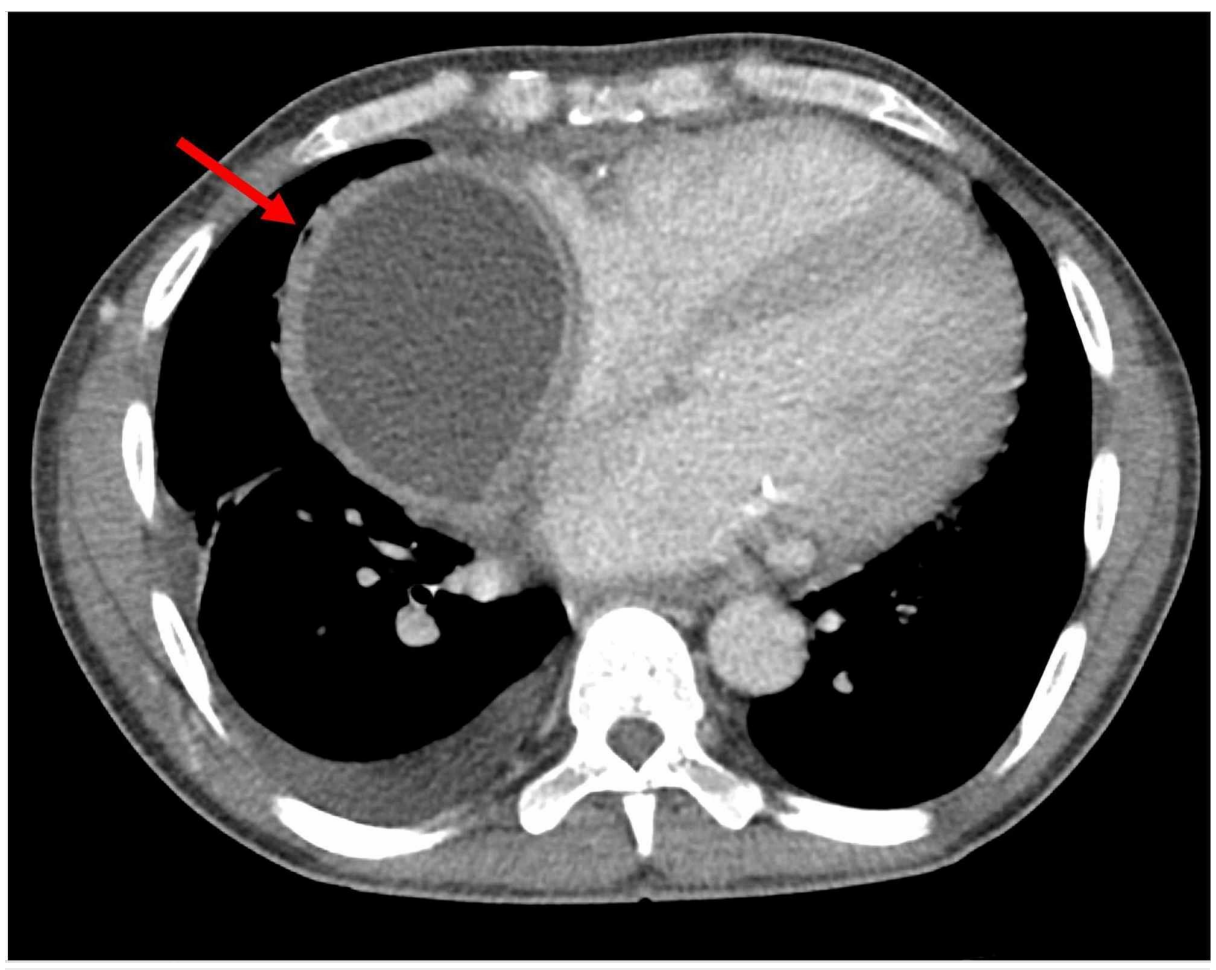

FIGURE 1: Pericardial Abscess: Axial CT Chest With IV Contrast. 


\section{Cureus}

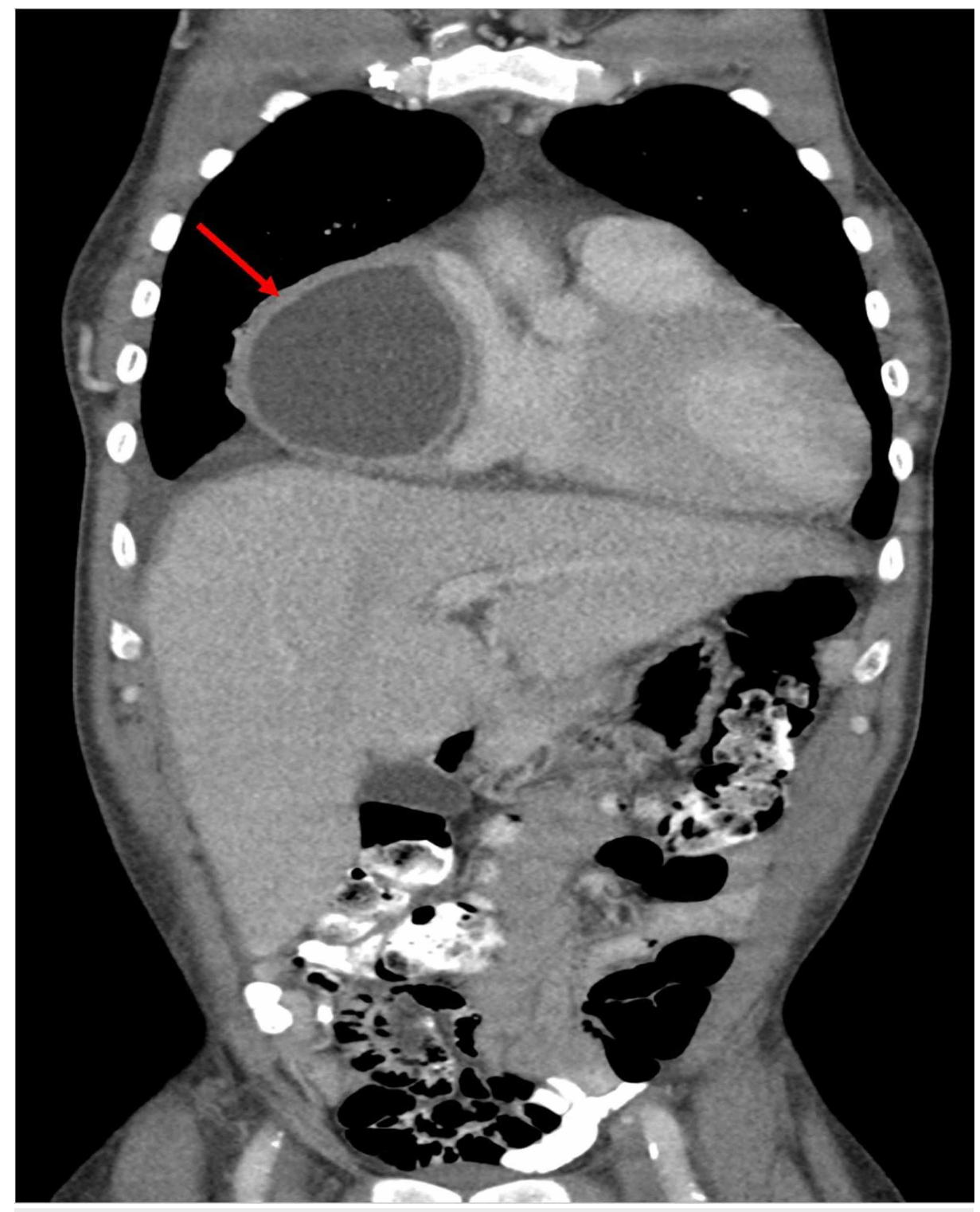

FIGURE 2: Pericardial Abscess: Coronal CT Chest, Abdomen, and Pelvis With IV and Oral Contrast. 


\section{Cureus}

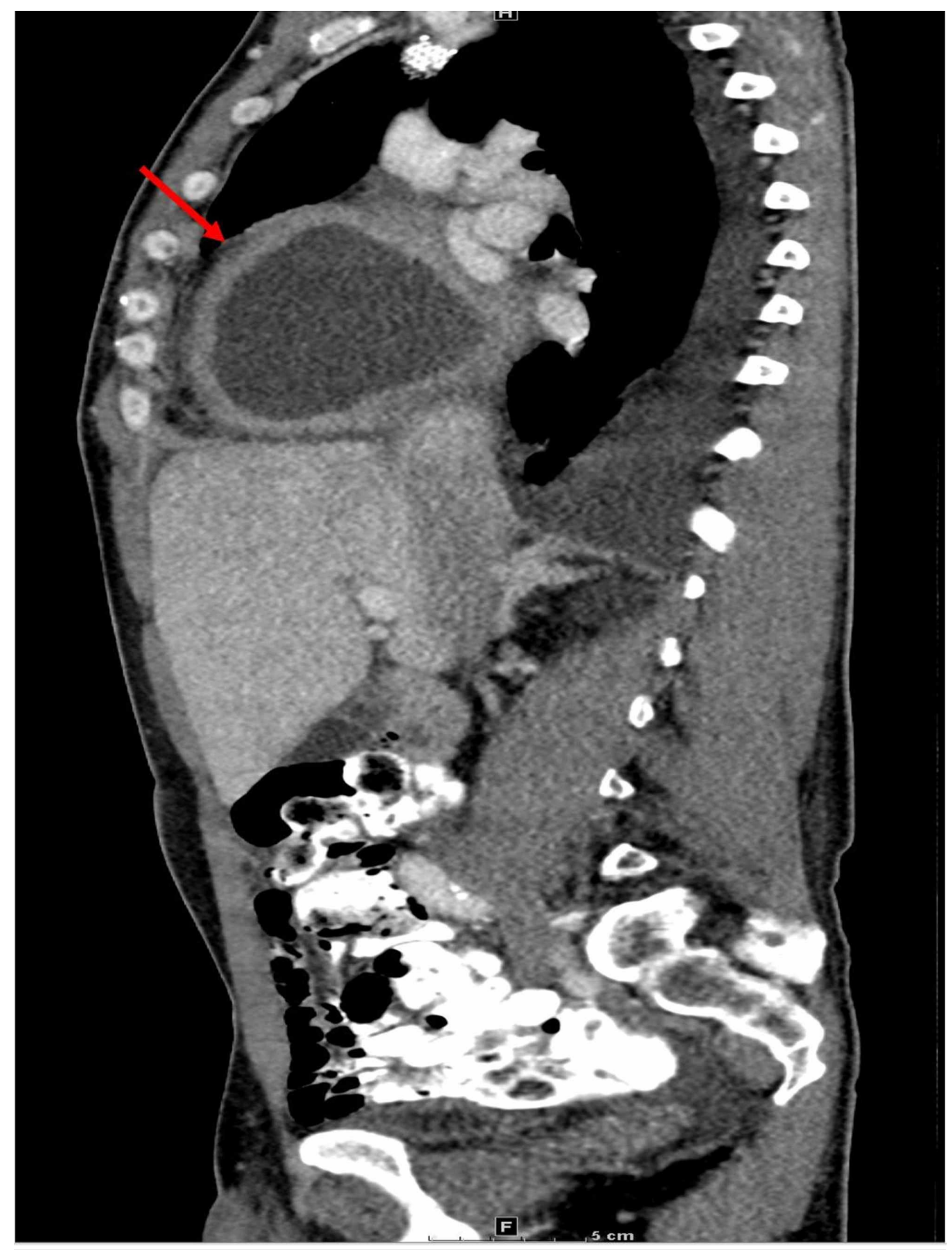

FIGURE 3: Pericardial Abscess: Sagittal CT Chest, Abdomen, and Pelvis With IV and Oral Contrast.

\section{Discussion}

Pericardial abscess has life-threatening consequences due to the risk of bacteremia and mass effect on the adjacent heart structures, resulting in severe hypotension [5]. The importance of recognizing such infections is dire; however, it can be challenging, as signs and symptoms of sepsis may seize to exist [5,6]. Therefore, radiology imaging and intervention becomes a crucial component for an accurate diagnosis to ensure immediate treatment. Pericardial masses are often initially detected with echocardiography, but CT and MRI can be warranted for further evaluation or characterization to aid in an accurate diagnosis of various pericardial masses [7].

Focal pericardial effusions, ventricular aneurysms, pericardial cysts, and fibromas all present mass effect on the heart contour, which is appreciated on radiological imaging [4]. The challenge then becomes differentiating a cyst from an infectious etiology, like an abscess, because cystic fluid and an abscess are similar in attenuation. In such a scenario, aspiration of the fluid with appropriate cytology and culture is required to differentiate the two $[8,9]$.

Pericardial mass is a rare etiology, and becomes even rarer with a superimposed Staphylococcus aureus infection, with very few other cases documented [4]. Our patient's history of ESRD managed with 
hemodialysis and two previous pericardial effusions secondary to uremic pericarditis are all risk factors that can lead to purulent pericarditis.

\section{Conclusions}

A pericardial abscess is a rare etiology affecting the heart and is considered a life-threatening complication. Prompt diagnosis with appropriate imaging, CT of the chest with intravenous contrast, is required to fully assess the nature of the disease process and to identify any aliment that may impact the patients stability. Definitive diagnosis is required by image-guided technique with fluid analysis. This allows for immediate implementation of therapy to ensure patient care.

\section{Additional Information}

\section{Disclosures}

Human subjects: Consent was obtained by all participants in this study. Conflicts of interest: In compliance with the ICMJE uniform disclosure form, all authors declare the following: Payment/services info: All authors have declared that no financial support was received from any organization for the submitted work. Financial relationships: All authors have declared that they have no financial relationships at present or within the previous three years with any organizations that might have an interest in the submitted work. Other relationships: All authors have declared that there are no other relationships or activities that could appear to have influenced the submitted work.

\section{References}

1. Sagristà-Sauleda J, Barrabés J, Permanyer-Miralda G, Soler-Soler J: Purulent pericarditis: review of a 20-year experience in a general hospital. J Am Coll Cardiol. 1993, 22:1661-1665. 10.1016/0735-1097(93)90592-O

2. Maisch B, Seferović P, Ristić A, et al.: Guidelines on the diagnosis and management of pericardial diseases executive summary: the task force on the diagnosis and management of pericardial diseases of the European Society of Cardiology. Eur Heart J. 2004, 25:587-610. 10.1016/j.ehj.2004.02.002

3. Sato TT, Geary RL, Ashbaugh DG, Jurkovich GJ: Diagnosis and management of pericardial abscess in trauma patients. Am J Surg. 1993, 165:637-641. 10.1016/S0002-9610(05)80451-9

4. Van de Donk NW, Meijer RC, America YG, Cramer MJ: Staphylococcus aureus pericardial abscess presenting as a localized bulge of the heart contour. Interact Cardiovasc Thorac Surg. 2010, 10:818-819. 10.1510/icvts.2009.230904

5. Wang ZJ, Reddy GP, Gotway MB, Yeh BM, Hetts SW, Higgins CB: CT and MR imaging of pericardial disease . Radiographics. 2003, 23:167-180. 10.1148/rg.23si035504

6. Kar SK, Ganguly T: Current concepts of diagnosis and management of pericardial cysts. Indian Heart J. 2017, 69:364-370. 10.1016/j.ihj.2017.02.021

7. Rubin RH, Moellering RC Jr: Clinical, microbiologic and therapeutic aspects of purulent pericarditis . Am J Med. 1975, 59:68-78. 10.1016/0002-9343(75)90323-x

8. Kenney RT, Li JS, Clyde WA Jr, et al.: Mycoplasmal pericarditis: evidence of invasive disease. Clin Infect Dis. 1993, 17:S58-S62. 10.1093/clinids/17.supplement_1.s58

9. Kraus WE, Valenstein PN, Corey GR : Purulent pericarditis caused by Candida: report of three cases and identification of high-risk populations as an aid to early diagnosis. Rev Infect Dis. 1988, 10:34-41. 10.1093/clinids/10.1.34 